\title{
Systemische Organisationsentwicklung im Krankenhaus Wie Chefärzte mit „ver-rückten“ Blickwinkeln Führungsvorbilder von morgen sein können
}

The people who are crazy enough to think they can change the world are the ones who do. Steve Jobs, "Think Different" Commercial, 1997

Ungeachtet des Leistungsdrucks des DRG-Systems und aller Zielvereinbarungsdiskussionen - die Hauptaufgabe des Chefarztes ist es, gute Medizin in seiner Klinik sicherzustellen und für die Zufriedenheit der Patienten zu sorgen. Dies ist in einem hocharbeitsteiligen Prozess zu bewältigen. Für die Arbeit an der dafür dienlichen Struktur, der Führung und den Abläufen in der eigenen Klinik nehmen sich Chefärzte und ihre leitenden Oberärzte in Abstimmung mit der kaufmännischen Leitung des Hauses oft erst Zeit, wenn wirklich dringender Handlungsbedarf aufgrund mangelnder Ressourcen oder ausbleibender Ergebnisqualität besteht oder wenn eine Neubesetzung der Chefarztposition ansteht. Mit Empathiefähigkeit für die Bedürfnisse und Möglichkeiten der Organisation und des Einzelnen sowie einem stimmigen Maß an Führungsimpulsen können Chefärzte Synergien aus ihren Teams ziehen. Immer wieder die richtige Balance in der Führung zu finden, fordert dabei echte Führungsqualitäten, um bewusstes Folgen aus Überzeugung zu erreichen. Und so kommen die „neuen Chefärzte“ mit ihren verrückten Blickwinkeln auf sich und ihr Umfeld auch der mit ihrer Position ursprünglich verbundenen Verantwortung wieder näher.

Sich im Alltag kontinuierlich mit der Organisation - gerade auch mit Unterstützung von außen - auseinanderzusetzen, wirkt zugegebenermaßen wenig attraktiv: Welcher Chefarzt möchte schließlich, dass seine Klinik, geschweige denn er selbst, entwickelt wird? Der betriebswirtschaftliche terminus technicus der „Organisationsentwicklung“" [1] ist da sperrig. Und doch ist klar: Die Entwicklung von Krankenhäusern und Großklinika funktioniert in Zeiten des patientenzentrierten DRG-Systems nicht ohne die Weiterentwicklung der „guten alten Welt" der Fachabteilungen. Über das Voranbringen zentraler Organisationsansätze und Querschnittsprozesse in der Versorgungskette hinaus ist das ein Plädoyer für die Weiterentwicklung der Führung in den fachspezifischen Verantwortungsbereichen und ihrer Schnittstellen zu anderen Bereichen. Wo sind die neuen Führungsvorbilder für heutige und künftige Chefärzte (und-ärztinnen ${ }^{2}$ ) in der Komplexität der heutigen netzwerkorientierten Zeit?

\section{Organisation: Struktur, Prozesse und Führung}

Ohne die Entwicklung von Führungskräften und ihrer Führungs- und Managementqualitäten unter den Anforderungen der modernen Krankenhauswelt kom- men Bemühungen in dem Bereich der Organisationsentwicklung von Fachabteilungen einem „Herumdoktern“ an den Symptomen gleich. Fachlich exzellenten Spitzenkräften sind gute Managementund Führungskompetenzen nicht automatisch in die Wiege gelegt. Auch wenn - nicht nur im Krankenhaus - jahrelang offenbar stillschweigend davon ausgegangen worden ist. Diese Ressourcen gilt es bewusst zu entfalten. Nicht zwingend müssen dann die Fähigkeiten oder Aufgabenzuordnungen zu Management und Führung in einer Person vereinigt sein. Denkbar ist durchaus, dass sich ein Chefarzt, der sich auf die Führung der Abteilung und die Behandlung der Patienten fokussieren möchte, ein Klinikmanagement oder einen geschäftsführenden Oberarzt zur Seite stellt. Eine zielgerichtete gemeinsame Organisation soll die Fachabteilung zum Blühen bringen.

\section{Führen heißt}

\section{vom Ende her zu denken}

Was meint eigentlich Management und Führung? Bekannte Größen der Betriebswirtschaft unterscheiden gar nicht zwischen den beiden Begriffen und in der Regel fallen die Aufgaben ja auch in ein und derselben Person zusammen. In der Organisationsentwicklung ist es allerdings hilfreich, beides zumindest gedank- lich zu unterscheiden. So ist es Aufgabe der Führung, Bilder der Zukunft in den Köpfen der Menschen zu entwickeln; Aufgabe des Managements ist es, diese in der Realität mit Leben zu füllen. Für Mitarbeiter ist es zumeist ausreichend, sich vertrauensvoll führen zu lassen und nur den vom Management vorgelegten nächsten Schritt im Blick zu haben.

John P. Kotter, der Papst des Changemanagements, beschreibt 1990 in seinem Buch „A Force For Change: How Leadership Differs From Management" den Unterschied zwischen Managern und wahren Führern („Leader“). Management stehe für das Organisieren der Abläufe, Planen und Kontrollieren. Leadership bedeute, andere mit Visionen zu inspirieren und zu motivieren. Leadership ist es also, was Kreativität, Innovation, Sinnerfüllung und Wandel schafft [2]. Management bringt die Vision zum Leben und pflegt die Strukturen, um so das Überleben zu sichern.

Wir sind mit Kotter der festen Überzeugung, dass effektive, synergetische Teams beide Typen als Führungsfiguren brauchen, obwohl Führungsbegabungen von Menschen selten in beide Richtungen zugleich gehen: Zu keiner Zeit können Organisationen ohne Manager auskommen, die alles gut zu organisieren verstehen. In Umbruchphasen ist gleichzeitig unbedingt ein Leader vonnöten, der die Menschen zusammenschweißen und auf eine neue Richtung einschwören kann. Dabei

\footnotetext{
${ }^{1}$ Organisationsentwicklung meint Interventionsstrategien, die gruppendynamische Prozesse in Organisationen verwenden, um geplante Veränderungen in Bezug auf Ergebnisziele herbeizuführen. Wesensmerkmal ist, dass sie auf einem mittel- bis langfristigen prozessorientierten, partizipativen Ansatz unter Berücksichtigung verhaltenswissenschaftlicher Erkenntnisse beruhen. Management von Organisationsentwicklungsprojekten wird häufig synonym als Organisationsentwicklung, Changemanagement bzw. Veränderungsmanagement bezeichnet. ${ }^{2}$ Im vorliegenden Text sind sämtliche Funktionen jeweils geschlechtsneutral gemeint.
} 
hat Leadership wenig mit bloßem Charisma zu tun. Die Kunst der Führung ist oft nüchterne, strategische Arbeit und bedarf vor allem eines - die Weitsicht, mit zunehmender Perspektive vom Ende her zu denken. Immer wieder situativ die richtige Balance zu finden und einen Weg zu gestalten, der visionäre Führung und auf Ordnung und Perfektion gerichtetes Management in einer Klinik miteinander integriert, ist ein Schlüssel zum Erfolg jeder Organisationsentwicklung.

Betrachtet aus einer Organisationsperspektive liegt ein Manager, der nach Ordnung und Perfektion in einer Klinik strebt, dem Bild des (Organisations-)Systems als eine Art Automat näher. Mit dem visionären Leader, der die Menschen mit auf den Weg nimmt und neue Perspektiven aufzeigt, verknüpft sich eher das Bild der Organisation als ein lebendes System.

Während die Organisation als Automat die Aufgabenspezialisierung und eine Standardisierung von Arbeitsprozessen in den Vordergrund stellt und stark auf einheitliche Vorschriften baut, um die Prozesse im Sinne einer hohen Effizienz und Sicherheit in den Abläufen steuerbar zu machen, fokussiert die Organisation als „lebendes und lernendes System“ auf die Komplexität im Zusammenspiel der Einzelnen und auf Weiterentwicklung. Dabei erhält das Zusammen- und Wechselspiel der einzelnen Einheiten eine eigene, wichtige Bedeutung. Die Idee, dass das System kontrollierbar ist, wird aufgegeben zugunsten einer Idee von Menschen in Lebensund Arbeitsprozessen, die sich teilautonom mit Entscheidungen einbringen, Ziele erkennen, kreativ und proaktiv an der Umsetzung und damit an der Weiterentwicklung der Organisation wirken [3].

\section{Systemische Organisationsentwicklung oder das} lebende System

Systemische Organisationsentwicklung schließt sich dem Bild der Organisation als lebendes System an. Das bedeutet nicht, dass die Beschreibungsqualität der Organisation als Automatismus mit seinen Stärken bedeutungslos wäre. Denn auch hier gilt es eine gesunde Balance herzustellen. Eine der wesentlichen Grundvoraussetzungen für das Fortbestehen einer Klinik ist, dass sie permanent an der Sicherheit und geringen Fehleranfälligkeit ihres Kernprozesses der Behandlung und Heilung von Menschen - arbeitet. Es braucht auf Sicherheit zugeschnittene, standardisierte Prozesse. Gleichzeitig fordert die Behandlung von Menschen ein hohes Maß an ganzheitlicher Betrachtung - was wäre da ein besserer Rahmen für das „lebende System“ Mensch als eine lebende Organisation? Zudem sind Kliniken Organisationen, denen verstärkt Anpassung und Weiterentwicklung abgefordert wird. Von innen heraus über Forschung und Lehre oder neue Behandlungsmethoden abgestimmt auf neue Erkenntnisse über das Funktionieren der Spezies Mensch. Von außen heraus durch Kostendruck, Einführung neuer Vergütungssysteme, gestiegene Anspruchshaltungen der Patienten, $\mathrm{Zu}$ nahme ambulanter Versorgungsformen und weitere Herausforderungen, die es zu meistern gilt.

Das „lebende System“ Organisation passt sich kontinuierlich an seine Umwelt an. Zu einem Großteil sind es externe Veränderungsimpulse, die den Druck auf die Organisation ausüben. Um das Positionieren und Überleben der Klinik si- 
cherzustellen, braucht es die permanente Auseinandersetzung mit den Bedürfnissen der Kunden und der Lage des Marktes. Gerade Fachabteilungen in Kliniken neigen dazu, zumindest in wichtigen Teilaspekten ihre Umwelt auszublenden. Dies trifft weniger auf die medizinischrelevanten Inhalte zu, denn hier sind insbesondere Ärzte stark fortschrittsorientiert. Geht es jedoch um Veränderungsimpulse, die eine Reflexionsfläche stärker in der Managementkompetenz und weniger in der Fachkompetenz benötigen, so entwickeln Kliniken oft einen blinden Fleck. Diesem blinden Flecken auf die Spur zu kommen und eine Organisations- und Führungskultur zu entwickeln, die das eigene Tun kontinuierlich im Abgleich mit Markt und Kunden sieht, ist ein entscheidender Schritt. Entscheidend ist die Wahrnehmung von Signalen, die heute noch schwach und unbedeutend, zukünftig jedoch erfolgsentscheidend sind. So hat die Dynamik der Einführung des DRG-Systems viele Kliniken unvorbereitet getroffen und notwendige Maßnahmen, um sich adäquat auf die damit verbundenen Veränderungen einzustellen, blieben aus. Dass solche Signale beachtet und gehört werden, dazu können alle Mitarbeiter beitragen. Doch so genannte Soundingboards oder ähnliche Instrumente findet man in Kliniken eher selten.

Ein weiterer wichtiger Grundsatz systemischer Organisationsentwicklung ist es, die Komplexität in ihren Neben-, Rückund Fernwirkungen zu erfassen und mögliche Wechselwirkungen in der Organisation und mit ihrer Umwelt prognostizierbar zu machen. Hier ein einfaches kleines Beispiel aus dem täglichen Klinikalltag: Auf einer Station einer großen Fachabteilung herrschte über einen längeren Zeitraum extremer Bettendruck und die Mitarbeiter arbeiteten an der Belastungsgrenze. Zusätzliche Ausfälle spitzten die Lage zu. Nach einer kurzfristig einberufenen Krisensitzung entschied sich der Chefarzt für einen Bettenstopp. In unmittelbarer Konsequenz darauf gingen am nächsten Tag die ersten Beschwerden niedergelassener Kollegen ein, die sich nun mit längeren Wartezeiten für ihre Patienten konfrontiert sahen und diese nicht akzeptieren wollten. Zudem meldete sich die Notaufnahme, die ohne den bisherigen Abfluss auf Station nun mit erhöhten Kapazitätsproblemen zu kämpfen hatte. Aufgrund der neuen
Probleme landete der Vorfall wieder auf dem Tisch des Chefarztes, der nun jedoch den Bettenstopp nicht ohne weiteres aufheben konnte, ohne eine Protest- und Verweigerungswelle seiner Mitarbeiter zu riskieren. Eine genaue Prüfung der Neben-, Rück- und Fernwirkungen hätte u.U. auch nicht zu einer anderen Entscheidung geführt, wohl aber zu einer besseren Kommunikation im Vorfeld und ggf. zu einem anderen Zeitplan für die Maßnahme. Vielleicht hätte aber auch die Einbindung von Niedergelassenen, Notaufnahme und anderen Beteiligten ganz andere Lösungsmöglichkeiten hervorgebracht.

\section{Systemisch führen und die Selbststeuerung stärken}

Dies bringt uns zur Stärkung oder Aktivierung der Selbststeuerungskraft in der Organisation. Mit diesem Ansatz setzt die systemische Organisationsentwicklung ein besonderes Führungsverständnis voraus: Stärkung der Selbststeuerung bedeutet, Management am System anstelle von Management im System. 3 Prinzipien [4] bilden dafür das Fundament:

- Betroffene beteiligen: Wer Betroffene beteiligt, gibt sich und den Betroffenen die Möglichkeit, ihr Wissen und ihre Fertigkeiten einzubringen und zu erproben. Das macht sie zu „Miteigentümern“ der Entwicklung - eine wichtige Voraussetzung dafür, dass sie persönlich Verantwortung übernehmen.

- Hilfe zur Selbsthilfe: Die Führungsaufgabe liegt im Anstoßen, der beratenden Unterstützung und Ermutigung. Der Mitarbeiter wächst an seinen Aufgaben im Bewusstsein, dass es keine Möglichkeit zur Rückdelegation gibt und er die Verantwortung trägt.

- Schrittweises Vorgehen: Es gilt das Prinzip des prozessorientierten Vorgehens. Es gibt zwar eine Soll-Konzeption, aber die Umsetzung erfolgt bewusst schrittweise, um aus jedem Schritt für den nächsten zu lernen. Schließlich bleibt das Umfeld in Bewegung und kann jederzeit Überraschungen bereiten.

Stärkung der Selbststeuerung bedeutet, dass Kliniken und Fachabteilungen so geführt werden, dass möglichst viele Mitarbeiter ihr Potenzial zur Selbstführung und Selbstverantwortung entfalten können und wollen.

\section{Neue Führungsvorbilder für} Chef- und ihre Oberärzte

Das Thema Weiterentwicklung ist heute in allen Lebensbereichen so schnell und präsent wie nie und machte ja selbst bei der Papstfrage oder in Königshäusern keinen Halt. Doch gerade in statusorientierten, veränderungsresistenten Organisationen scheinen schnelle und leicht empfundene Organisationsentwicklungen fast noch außerhalb des Denkbaren zu liegen. Die Führungsebene prägt meistens noch ein Heldenmythos, der alles auf einen allein Verantwortlichen ausrichtet, der persönlich die Führung übernimmt. Ein Bild, das viele Jahrzehnte oder gar Jahrhunderte lang in Kliniken durch das Bild vom „Mediziner als Gott in Weiß“" gestützt wurde. Und auch wenn sich zwischenzeitlich das Bild gewandelt hat, so schimmern die historischen Wurzeln in der Klinikorganisation immer noch durch. Denn oft findet sich bei Patienten und auch Mitarbeitern die Sehnsucht nach dem Chefarzt als haltgebenden Helden, der alles richten kann. Eine klare Ordnung und Hierarchie geben Halt und Stabilität gerade in unsicheren Zeiten und Krisensituationen des Lebens.

Doch was bedeutet dieser Heldenmythos angesichts der Grundprinzipien systemischer Führung? Sind die Führungsimpulse in einer Klinik zu stark, werden der Selbststeuerungswille und die Selbstverantwortung der Mitarbeiter geschwächt. Sind die Führungsimpulse in der hierarchischen Ordnung allerdings zu schwach, verharren die Mitarbeiter möglicherweise in ihrer inneren Komfortzone und richten sich eher nach ihren Interessen denn nach den Interessen des Unternehmens aus. Selbststeuerung und Selbstverantwortung werden erst gar nicht angeregt. Das richtige Maß zu finden ist eine sich immer wieder neu stellende Herausforderung.

Es gibt sie heute schon: Die Chefärzte und durchaus auch solche, die schon lange in Amt und Würden sind -, die sich an der mit vereinter Intelligenz ihrer Abteilung gemeinsam erreichten Sache erfreuen und sich vom Heldenstatus verabschieden. Mit Empathiefähigkeit für die Bedürfnisse und Möglichkeiten der Organisation und des Einzelnen sowie einem stimmigen Maß an Führungsimpulsen holen sie Synergien aus ihren Teams heraus. Und so kommen sie auch der mit ihrer Position ursprünglich verbundenen Verantwortung wieder näher: Tatsäch- 
lich leitet sich die Hierarchie - bereits in der frühen christlichen Kirche [5] - nicht aus Ober- und Unterordnung ab, sondern aus einer Ordnung im Sinne eines Verantwortungssystems, in der jeder seine verschiedenen Begabungen einbringt und damit dem Ganzen nach den eigenen Möglichkeiten dient. Immer wieder die richtige Balance in der Führung zu finden, fordert dabei echte Führungsqualitäten, um bewusstes Folgen aus Überzeugung zu erreichen. Es müssen andere Grenzziehungen erlernt werden, etwa um nichtförderliche informelle Machtstrukturen und Konflikte im Team aufzulösen und Fragestellungen zu klären, die durch Ausnutzen der neuen Freiheiten im systemischen Organisationsgefüge erwachsen können.

Wenn diese „neuen Chefärzte“ mit ihren „ver-rückten“ Blickwinkeln beständig trotz schwieriger Entwicklungsphasen an sich und ihre Idee von Organisation glauben, dann werden sie vielleicht die Führungsvorbilder von morgen sein, die die Welt der Kliniken nachhaltig verändern.

\section{Literatur}

1 Christopher Mabey, Derek S. Pugh. Strategies for Managing Complex Change. The Open University, Milton Keynes, 1999

2 Harvard Business Manager, Heft 4/2004. Führung. Ganz neu war diese Differenzierung nicht, sondern es gab bereits Unterscheidungen wie etwa zwischen transaktionaler und transformativer Führung nach James M. Burns

3 R. Henning, G. Strina, Systemische Organisationsberatung. In: Handbuch der Unternehmensberatung, Band 1. Berlin 2010; Erich Schmidt Verlag GmbH \& Co KG: 3215 ff

4 Dr. Klaus Doppler. Über Helden und Weisen - von heldenhafter Führung im System zu weiser Führung am System. In: OrganisationsEntwicklung 2009; Nr. 2: 4-12

5 Philipperbrief 1,1, oder 1 Korintherbrief Kap. 12 (Danke an Dr. von Zedtwitz für den interessanten Hinweis)

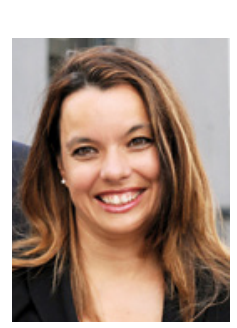

Korrespondenz

Dr. Elke Eberts

Ruhl Consulting AC

Harrlachweg 1

68163 Mannheim

Tel.: 0621/32886490

E-Mail: info@ruhl-

consulting.de

Internet: www.ruhl-

consulting.de

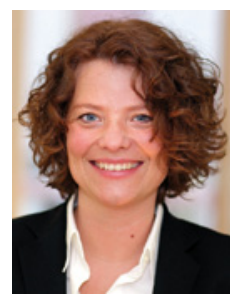

Nicole Krüttgen

Gestaltungszeit

Tornquiststr. 77

20259 Hamburg

Tel.: 040/79306536

E-Mail: info@gestaltungszeit.de www.gestaltungszeit.de 